\title{
Measurements of the CKM Sides at the B Factories
}

\author{
Isamu Nakamura
}

KEK, 1-1 Oho Tsukuba Ibaraki, 305-0801, Japan

\begin{abstract}
Recent results of the measurements of the CKM sides at B factories are summarised. In this talk, both inclusive and exclusive measurements of the CKM matrix elements $V_{\mathrm{ub}}$ and $V_{\mathrm{cb}}$, carried out by two experiments BaBar and Belle, are presented.
\end{abstract}

\section{Introduction}

After the discovery of CP violation in B decay, the theory of CKM has been tested extensively in B factories. So far all measurements are consistent with the theory of CKM. To go further one has to improve the accuracy of the measurements in the angles and sides of the CKM triangle. Among these measurements the angle $\beta$ (or $\phi_{1}$ ) is most precisely determined and its error is a mere one degree. Since the side $\left|V_{\mathrm{ub}} / V_{\mathrm{cb}}\right|$ is opposite to the angle $\beta$, and is less accurately measured compared to $\beta$, improvement of the measurement of the side $\left|V_{\mathrm{ub}} / V_{\mathrm{cb}}\right|$ is very important to verify further the theory of CKM. Figure 1 shows the allowed region of the CKM triangle determined by measurements of angles(left) and sides(right) only.
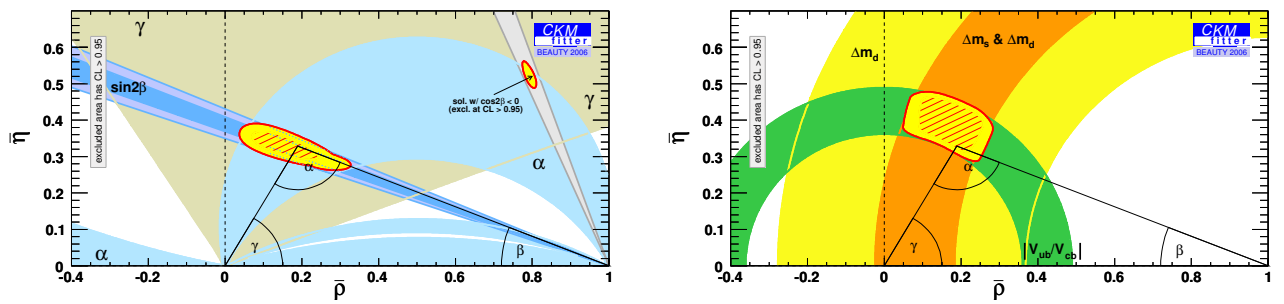

Figure 1: Allowed region of the CKM triagnle.

The CKM element $V_{\mathrm{xb}}$ can be determined using semileptonic decays given by the formula,

$$
\Gamma\left(\mathrm{b} \rightarrow \mathrm{x} \ell^{-} \bar{\nu}\right)=\frac{G_{F}^{2}}{192 \pi^{2}}\left|V_{\mathrm{xb}}\right|^{2} m_{\mathrm{b}}^{5}(1+\text { C.F. }),
$$

where $\mathrm{x}$ is either $\mathrm{c}$ or $\mathrm{u}$. So basically $V_{\mathrm{xb}}$ can be determined by counting the number of $\mathrm{b} \rightarrow \mathrm{x} \ell \nu$ events. However for the precise measurement, determination of the correction term, C.F., is necessary, and is known to be difficult. In the inclusive measurement the term is determined with the help of Heavy Quark Effective theory (HQET), while in the exclusive measurement it is determined in terms of form factor by Lattice QCD.

All averaged results are taken from the Heavy Flavour Averaging Group [2]

\section{Measurement of $V_{\mathrm{cb}}$}

\section{1 $V_{\mathrm{cb}}$ from inclusive semileptonic decays}

For the inclusive decay $\mathrm{b} \rightarrow \mathrm{c} \ell \nu$, the C.F. in Equation 1 is described as

$$
\text { C.F. }=\left(1+A_{\text {ew }}\right) A_{\text {nonpert }} A_{\text {pert }}=f_{\mathrm{OPE}}\left(m_{\mathrm{b}}, m_{\mathrm{c}}, a_{i}\right) \text {, }
$$


where $m_{\mathrm{b}}, m_{\mathrm{c}}$ are the masses of the $\mathrm{b}$ and c quark and $a_{i}$ are additional parameters depending on the scheme. There are two separate calculations available for the function $f_{\mathrm{OPE}}$, in the kinetic scheme [3] and the 1S scheme [3]. In these calculations, distributions of experimental observables, lepton momentum and invariant mass of the hadronic part of the decay, are predicted. By fitting these distributions, $f_{\mathrm{OPE}}$, and hence $V_{\mathrm{cb}}$ can be determined.
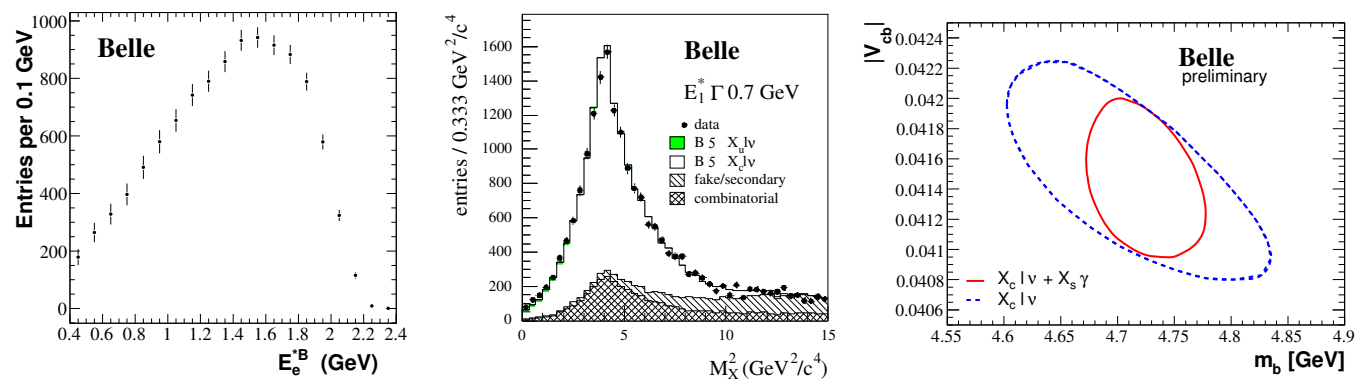

Figure 2: Lepton momentum (left), Hadronic mass (center), and $V_{\mathrm{cb}}-m_{\mathrm{b}}$ contour (right)

Figure 2 shows the lepton momentum (left) and hadronic mass distribution (center) measured by Belle [4] as well as the fitted $V_{\mathrm{cb}}-m_{\mathrm{b}}$ contour (right) in the kinetic scheme. $V_{\mathrm{cb}}$ is determined as $(41.93 \pm 0.91) \times 10^{-3}$ and $(41.49 \pm 0.56) \times 10^{-3}$ for the kinetic and $1 \mathrm{~S}$ scheme, respectively $[2]$.

\section{$2.2 \quad V_{\mathrm{cb}}$ from exclusive semileptonic decays}

The CKM parameter $V_{\mathrm{cb}}$ is also measured by utilizing an exclusive decay $\mathrm{B} \rightarrow \mathrm{D} * \ell \nu$ with the following formula,

$$
\frac{d \Gamma\left(\mathrm{B} \rightarrow \mathrm{D}^{*} \ell \nu\right)}{\mathrm{d} w \mathrm{~d} \cos \theta_{\ell} \mathrm{d} \cos \theta_{V} \mathrm{~d} \chi}=\frac{G_{F}^{2}}{48 \pi^{3}} \mathcal{G}(w)\left|V_{\mathrm{cb}}\right|^{2} \mathcal{F}\left(w ; \cos \theta_{\ell}, \cos \theta_{V}, \chi, f_{j}\right),
$$

where $w$ is the boost of the $\mathrm{D}^{*}$ in the $\mathrm{B}$ restframe, $\theta_{\ell}, \theta_{V}, \chi$ are angles of the decay products defined in Figure 3 and $f_{j}$ are the form factors. BaBar measured three angular distributions to fit simultaneously the form factor $f_{j}$ and $w$ distributions using data corresponding to $79 \mathrm{fb}^{-1}[5]$. Figure 3 shows also the distribution of $w$. By averaging the result with the
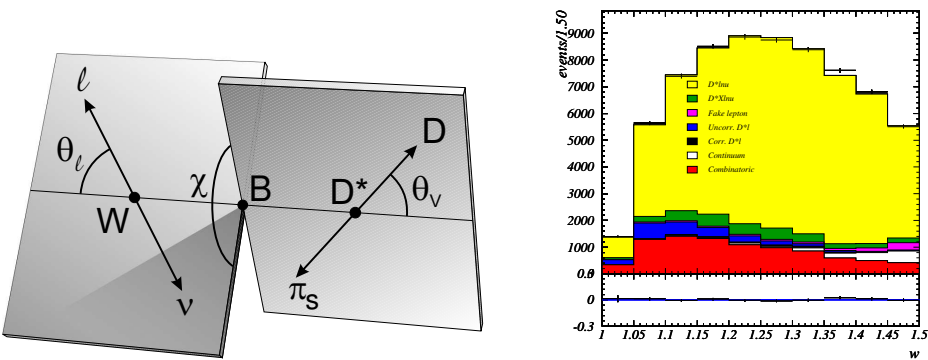

Figure 3: Definition of angles (left), $w$ distribution (right)

other measurements, $\mathcal{F}(1)\left|V_{\text {cb }}\right|$ is determined as $(35.8 \pm 0.6) \times 10^{-3}$. Using the lattice QCD calculation of $\mathcal{F}(1)=0.919_{-0.035}^{+0.030}[6]$, a $V_{\text {cb }}$ value of $\left(39.0 \pm 0.7_{-1.5}^{+1.3}\right) \times 10^{-3}$ is obtained. 


\section{Measurement of $V_{\mathrm{ub}}$}

\section{1 $V_{\mathrm{ub}}$ from inclusive semileptonic decays}

The CKM element $V_{\mathrm{ub}}$ can be determined by measuring the inclusive branching fraction $\mathrm{B}\left(\mathrm{B} \rightarrow \mathrm{X}_{\mathrm{u}} \ell \nu\right)$ using equation 1 . Since the $\mathrm{b} \rightarrow \mathrm{u}$ branching fraction is about 50 times smaller than that of $\mathrm{b} \rightarrow \mathrm{c}$, one has to utilize some kinematic variables, such as the lepton momentum, the leptonic invariant mass, $q^{2}$, or the hadronic mass, $M_{\mathrm{X}}$, to enhance $\mathrm{b} \rightarrow \mathrm{u}$ decay against $\mathrm{b} \rightarrow \mathrm{c}$. Hence, what is actually measured is the partial branching fraction. From the partial branching fraction, $V_{\mathrm{ub}}$ can be determined by the formula,

$$
\left|V_{\mathrm{ub}}\right|=\sqrt{\frac{\Delta \mathrm{B}\left(\mathrm{b} \rightarrow \mathrm{u} \ell^{-} \bar{\nu}\right)}{\mathcal{R}(\Delta \phi)}},
$$

where the factor $\mathcal{R}(\Delta \phi)$ is the partial rate in the kinematic phase space predicted by theory. There are several calculations available for this factor $\mathcal{R}$. Figure 4 shows the $q^{2}$ distribution
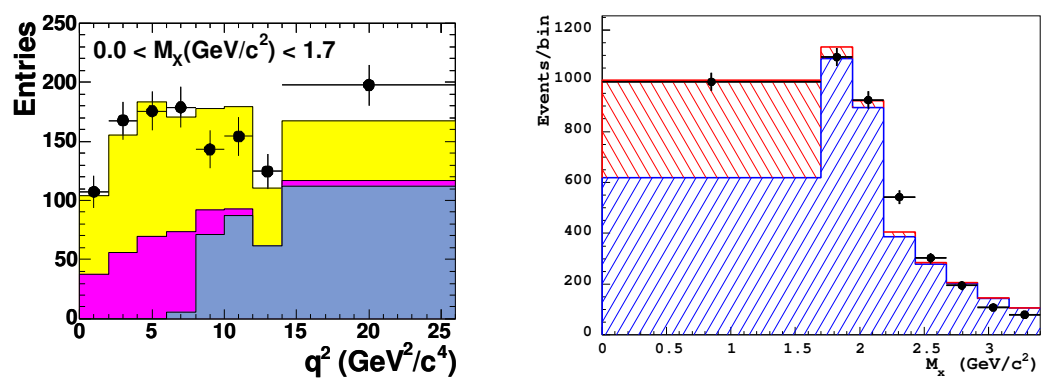

Figure 4: $q^{2}$ distribution (left) and hadronic mass distribution (right)

(left) measured by BaBar [7] and the distribution of hadronic mass (right) measured by Belle [7]. Fitting these distributions with the expected $b \rightarrow u$ and $b \rightarrow c$ shape, the number of $\mathrm{b} \rightarrow \mathrm{u}$ events, hence the partial branching fractions are obtained. The measured branching fractions with various kinematic selections are averaged and turned into a $V_{\mathrm{ub}}$ value using $\mathcal{R}$ predicted by theory. For the BLNP calculation $[8]\left|V_{\mathrm{ub}}\right|=\left(4.52 \pm 0.19_{\exp } \pm 0.27_{\mathrm{th}}\right) \times 10^{-3}$ and for the DGE calculation $[8]\left|V_{\mathrm{ub}}\right|=\left(4.46 \pm 0.20_{\exp } \pm 0.20_{\mathrm{th}}\right) \times 10^{-3}$.

\section{2 $\quad V_{\mathrm{ub}}$ from exclusive semileptonic decays}

The CKM element $V_{\mathrm{ub}}$ can be measured in the exclusive decay $\mathrm{B} \rightarrow \pi \ell \nu$ using the differential rate, given by

$$
\frac{d \Gamma(\mathrm{B} \rightarrow \pi \ell \nu)}{\mathrm{d} q^{2}}=\frac{G_{F}^{2}\left|V_{\mathrm{ub}}\right|^{2}}{192 \pi^{2} m_{\mathrm{b}}^{3}} \lambda\left(q^{2}\right)^{\frac{3}{2}}\left|f\left(q^{2}\right)\right|^{2}, \quad q^{2}=\left(p_{\ell}+p_{\nu}\right)^{2} .
$$

The form factor $\left|f\left(q^{2}\right)\right|$ is calculated by theories, such as Light Cone Sum Rules [9] or Lattice QCD [9]. Experimentally, there are three different analyses for three different tagging methods. The analysis without requirement on the other side of the $\mathrm{B}$ has an advantage in the event statistics, while it suffers from large number of background events. On the 
other hand the tagged analysis has smallest efficiency with purest signal, which is shown in Figure 5 (Left) [10], where the signal can be clearly separated from the background. Also shown in the Figure is the $q^{2}$ distribution obtained in BaBar's untagged analysis as well as the fitted distribution predicted by various theories [10]. By averaging the results of the measurements, $\left|V_{\mathrm{ub}}\right|$ is calculated as $\left(3.41 \pm 0.12_{\exp }{ }_{-0.38}^{+0.56} \mathrm{th}\right) \times 10^{-3}$ using the form factor calculated by LCSR [9] and $\left(3.97 \pm 0.22_{\exp }{ }_{-0.41}^{+0.59}\right.$ th $) \times 10^{-3}$ using unquenched lattice QCD calculation [9].
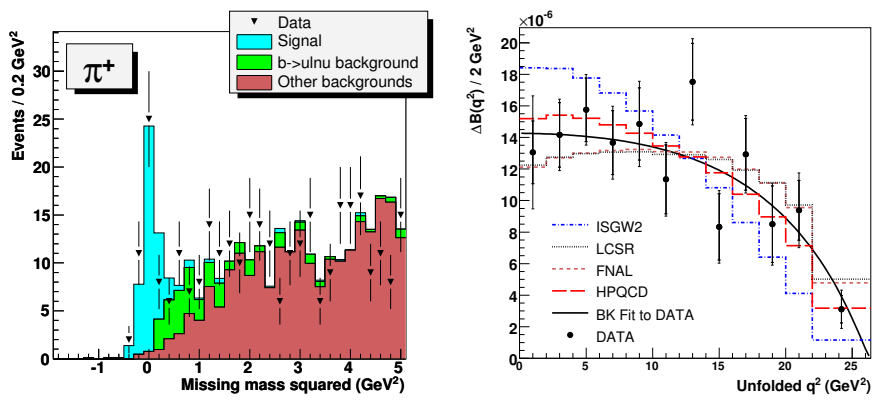

Figure 5: Reconstructed neutrino mass (left) and $q^{2}$ (right) distribution

\section{Summary}

The CKM elements $V_{\mathrm{cb}}$ and $V_{\mathrm{ub}}$ are measured using both inclusive and exclusive decays. The results are summarised in Table 1. Although these results are obtained by different experimental techniques and using different theoretical treatments, the re-

\begin{tabular}{|c|c|c|}
\hline & $\left|V_{\mathrm{cb}}\right|\left(10^{-3}\right)$ & $\left|V_{\mathrm{ub}}\right|\left(10^{-3}\right)$ \\
\hline Exclusive & $39.4 \pm 1.6$ & $4.0 \pm 0.6$ \\
\hline Inclusive & $41.5 \pm 0.6$ & $4.5 \pm 0.3$ \\
\hline
\end{tabular}

Table 1: Measured values of $V_{\mathrm{cb}}$ and $V_{\mathrm{ub}}$ sults are very consistent with each other.

Some measurements are limited experimentally and some are by the theory uncertainty. To get the precision of $5 \%$, both experimental and theoretical effort are necessary.

\section{References}

[1] Slides: http://indico. cern. ch/contributionDisplay py? contribId=114\&amp; sessionId=9\&amp; conf Id=9499

[2] HFAG Summer2006, E. Barberio et al., arXiv:0704.3575

[3] 1S: C. Bauer, et al., Rhys. Rev. D70 094017; Kinetic: P. Gambino, et al., Eur. Phys. J C34 181

[4] C. Schwanda, et al., Rhys. Rev. D75 32005(2007); P. Urquijo, et al. Rhys. Rev. D75 32001(2007)

[5] BaBar Collaboration, hep-ex/0607076.

[6] S. Hashimoto, et al., Phys. Rev. D66 014503(2002)

[7] BaBar Collaboration, hep-ex/0507017; Belle Collaboration, I. Bizjak et al., Rhys. Rev. Lett 95241801 2005

[8] BLNP: S.W. Bosch, et al., Phys. Rev. D72 073006(2005); DGE: J.R. Anderson, et al., JHEP, 061:097(2006)

[9] LCSR: P. Ball, et al., Phys. Rev. D71 014029(2005); LQCD: E. Dalgic,et al.,Phys. Rev. D73 074502(2006)

[10] BaBar Collaboration, hep-ex/0612020; Belle Collaboration, hep-ex/0610054 